\title{
Influencia de la experiencia de marca en consumidores de compras online en la ciudad de Cuenca-Ecuador
}

\section{Influence of the brand experience on online shopping consumers in the city of Cuenca-Ecuador

\author{
Marco Vinicio Moscoso Merchán, Carlos Iván Piña Cortez², María Fabiola Saquicela \\ Cobos $^{3}$
}

1. Ingeniero en Administración, profesor Instituto Tecnológico Superior Particular Sudamericano Cuenca - Ecuador. Email: mmoscoso@sudamericano.edu.ec ORCID: https://orcid.org/0000-0001-9115-5742

2. Ingeniero Comercial, profesor Instituto Tecnológico Superior Particular Sudamericano Cuenca - Ecuador. Email: carpico@sudamericano.edu.ec ORCID: https://orcid.org/0000-0001-7976-4561

3. Magister en Auditoría Integral y Gestión de Riesgos Financieros, profesora Instituto Tecnológico Superior Particular Sudamericano Cuenca Ecuador. Email: mfsaquicela@sudamericano.edu.ec ORCID: https://orcid.org/0000-0003-3879-1429

Para Citar: Moscoso Merchán, M. V., Piña Cortez, C. I., \& Saquicela Cobos, M. F. (2020). Influencia de la experiencia de marca en consumidores de compras online en la ciudad de Cuenca-Ecuador. Revista Publicando, 7(25), 146-159. Recuperado a partir de https://revistapublicando.org/revista/index.php/crv/article/view/2098

Resumen: En este artículo se busca analizar la influencia que tiene la experiencia de marca en los consumidores de compras on-line en la ciudad de Cuenca - Ecuador. En la investigación se ejecutará un análisis documental de las principales teorías del "marketing experiencial", revisando las fuentes bibliográficas para ir delimitando el marco referencial que constituye las bases teóricas del estudio, el cual pretende generar una visión holística de la influencia de la experiencia de marca en el comportamiento del consumidor de compras on-line. Se ha considerado iniciar con la identificación de las líneas de investigación centradas en el análisis y estudio del comportamiento del consumidor y la relación directa que éstos tienen con las "experiencias" que generan las tiendas electrónicas. Además, para llevar a cabo la investigación se plantea un enfoque metodológico que permite enmarcar la investigación para la obtención de resultados y con ellos brindar una orientación aplicativa para el sector académico y profesional. La finalidad de la investigación es aportar por un lado al campo académico mediante la incorporación de conocimientos en los estudiantes, fortaleciendo así sus competencias; además, al sector productivo y profesional de Cuenca y su región como una herramienta concluyente e identificativa referente a las nuevas tendencias en los hábitos de compra de los consumidores locales.

Palabras clave: Marketing experiencial, experiencia de marca, investigación, influencia de la experiencia,
Abstract: In this article it is analyzed the influence of brand experience on consumers of online shopping in the city of Cuenca - Ecuador. The research will carry out a documentary analysis of the main theories of "experiential marketing", reviewing the main bibliographic sources to delimit the referential framework that will constitute the theoretical bases of the study. It aims to generate a holistic vision of the influence of brand experience on consumer behavior of online shopping. It has been considered to start with the identification of lines of research focused on the analysis and study of consumer behavior and the direct relationship that consumers have with the "experiences" generated by electronic stores. In addition, in order to carry out the research, a methodological approach is proposed that allows research to be framed in order to obtain results and with them to be able to provide an application orientation for the academic and professional sector. The aim of the research is to contribute on the one hand to the academic field by incorporating knowledge in students, thus strengthening their competencies; the productive and professional sector of Cuenca and its region as a conclusive and identifying tool regarding the new trends in the purchasing habits of local consumers.

Keywords: Experiential marketing, branding experience, research, experience influence, consumer behavior. 


\section{INTRODUCCIÓN}

$\mathrm{E}$ la actualidad nos hallamos atravesando una revolución, en la que hemos dejado de lado el enfoque tradicional del marketing de características y ventajas por experiencias de clientes. Este nuevo enfoque influirá directamente en la manera de hacer negocios. En este sentido Schmitt sostiene que la "omnipresencia de la tecnología de la información" donde la tecnología ha buscado la forma de implantarse dentro de la vida de las personas. Es por ello, que se evidencia un acelerado progreso tecnológico, facilitando la interacción de las personas desde cualquier parte del mundo. Esto permitirá el nacimiento de un universo experiencial, como una nueva manera de satisfacción (Schmitt, 2000).

El marketing tradicional se orienta a las características y ventajas funcionales. Kotler define a las características como "peculiaridades que complementan la función básica del producto" y justifica como los clientes seleccionan los productos basándose en características (Kotler \& Keller, 2006).

Mientras tanto Michael Porter refiere la diferenciación de los productos, dentro del entorno competitivo como el alcance de una posición especial en un atributo que sea "ampliamente valorado por los consumidores". Es oportuno manifestar que las ventajas se derivan de las características funcionales (Porter, 2015).

La metodología de investigación desde la óptica del marketing tradicional se centra en análisis de datos objetivos y numéricos que aportan información estadística del consumidor, pero carente de un fundamento psicológico y de la manera que los clientes perciben los productos.

Con esta visión no se toma en consideración la esencia de la marca, y cómo ésta aporta en el comportamiento del consumidor, a través de asociaciones sensoriales, afectivas y cognitivas que dan como resultado una experiencia significativa y memorable. Los símbolos, diseños, herramientas como logos y nombres ya no tienen el mismo efecto que en el pasado, ya no activan la elección del cliente en muchos niveles.

En la actualidad los clientes desean respuestas más efectivas, soluciones innovadoras y marcas con las que se puedan conectar. Por tanto, el marketing experiencial no está supeditado a un solo modelo metodológico, sino más bien se considera ecléctico, es de carácter indagador, principalmente en cuatro aspectos: se enfoca en las experiencias del consumidor, gestiona el consumo como una experiencia holística, identifica los impulsores de compra tanto racionales como emocionales del consumo y utiliza metodologías eclécticas.

Un creciente número de empresas de éxito, en la actualidad han descubierto las virtudes del enfoque experiencial, y es así que marcas como Microsoft acude a herramientas del marketing experiencial, diseñando campañas orientadas a experiencias del pensamiento e imaginación, mediante anuncios impresos plantean la incógnita ¿Dónde desea ir usted después? esta campaña gira en torno a al potencial y los sueños, y no a ventajas y características.

Así mismo, observamos que la marca había venido desarrollando el tópico del marketing de identidad, colocando su sello "Intel Inside" sin embargo, sus más recientes acciones, se orientan más al campo sensorial con un "eslogan auditivo" de Intel, un tono formado por cuatro notas y así podríamos citar también a Windows y Macintosh que utiliza estas mismas herramientas.

Nos referimos a la experiencia como sucesos que responden a una estimulación, por lo general no son innatas sino de carácter inducido. Existen cinco tipos de experiencias que conforman el marco del experiential marketing (Schmitt, 2000).

a) Sensorial: se orienta a los sentidos con la finalidad de crear experiencias sensoriales. Se lo utiliza para diferenciar, productos y empresas, para entregar un valor agregado.

b) Sentimientos: apela a las emociones y sentimientos de los clientes, con el fin de crear experiencias afectivas, que van desde estados de ánimo hasta fuertes vínculos con la marca. Para que esto sea efectivo, se requiere provocar estímulos y la disposición de los consumidores. 
c) Pensamiento: Ofrece experiencias cognitivas, a través del intelecto resolviendo problemas y atrayéndolos creativamente. Los pensamientos apelan a la atracción del pensamiento convergente y divergente de los clientes por medio da la sorpresa.

d) Actuaciones: promueve la interacción, enriquece la vida de los clientes mostrándoles a través de alternativas, nuevas maneras de hacer las cosas.

e) Relaciones: Contienen aspectos emocionales, de sentimientos, pensamientos y sensoriales.

Estos 5 tipos de experiencias constituyen los Módulos Estratégicos Experienciales (MEES), los mismos que se producen por medio de:

a) Publicidad: catálogos, informes anuales.

b) Identidad. Logotipos, nombres, símbolos.

c) Presencia del producto: personajes de marca, empaques.

d) Cogestión de marcas: eventos y patrocinios, aparición de productos.

e) Entornos espaciales

f) Páginas web - medios electrónicos.

g) Personal.

Además, podemos indicar también que, una de las propuestas de valor de Lindstrom (2012), es crear un marketing que apele a los 5 sentidos, manifiesta que la marca debe ser identificable sin su logo, Un marketing que abarque los cinco sentidos debe prestar atención a los siguientes aspectos del producto.

- En cuanto a la vista, el logo, el diseño del producto, el color o colores y el tipo de letra.

- Para el sonido, la música y los sonidos del producto.
- El sabor del producto y los obsequios comestibles apelan al gusto; y el aroma ambiental y del producto, al olor.

- Y para crear una impresión táctil de su producto, preste atención a su superficie y su forma, así como a la textura de los materiales de marketing y a las superficies ambientales (Lindstrom, 2012).

Para el desarrollo de esta investigación debemos primeramente conocer cómo se comporta el consumidor ecuatoriano en el entorno online, por eso es fundamental identificar qué es lo que realizan o cuál es el proceso de compra que utilizan y esto lo vamos a sustentar mediante el primer estudio del comportamiento del consumidor electrónico en Ecuador, que fue realizado mediante la iniciativa de la Cámara Ecuatoriana de Comercio Electrónico (CECE) (Electrónico, 2017).

Hombres ecuatorianos de 26 a 33 años serían los que más compran por Internet, siendo la categoría de vestimenta la sección que más ventas online tiene en Ecuador, Según dicha investigación realizada entre agosto y septiembre del 2017 a más de 1.284 personas que residen en el territorio ecuatoriano, cerca del $92 \%$ realiza una compra o servicio online al día, tales como pago de transacciones bancarias o búsqueda de información, de acuerdo con datos proporcionados por Diario El Universo (2017).

Asimismo, de las personas encuestadas un 76\% realiza una adquisición en portales extranjeros, los artículos electrónicos, medicamentos y libros, son otros de los bienes no personales con más demanda en Internet en Ecuador, seguido de vestimenta y cosmética, siendo los portales más solicitado para esas compras Amazon y De Prati.

Otros de los datos reveladores del estudio, es que, si bien los usuarios en Ecuador usan un dispositivo móvil para buscar información, la mayoría opta por efectuar su transacción desde una computadora fija o laptop.

La investigación también resaltó que aún persiste el miedo y la desconfianza por parte de los ecuatorianos de comprar a través de una página web. 
Los ecuatorianos encuentran en esta forma de hacer negocios por Internet varias razones que hacen que este hábito crezca entre los internautas:

- Contar con garantía de devolución y/o cambio: $53 \%$

- Garantía de confidencialidad de la información: $41 \%$

- Más información de cómo comprar: $29 \%$

- Atención al cliente durante la compra: $26 \%$

Entre otros datos que se obtuvieron está ¿Qué compran los ecuatorianos? Y los resultados dieron la siguiente información:

- Servicios: es la categoría de mayor crecimiento, los pasajes son el rubro más comprado por todos los grupos etarios, seguido por los hoteles en los rangos etarios de adultos.

- Bienes no personales: Dispositivos electrónicos y todo lo referente a computación son los bienes de más consumo. En general son los hombres los que más compran dentro de esta categoría, que además alberga mayor compra por personas mayores de los 50 años.

- En esta misma categoría de Bienes: los hombres compran más ropa, mientras que las mujeres suman a sus compras más accesorios y cosméticos. La mayor proporción de compradores online se concentra entre los 26 y 33 años.

- Productos para el hogar: es la categoría de menor frecuencia de compra vía Internet, no se detectaron diferencias significativas por género. Muebles, decoración y productos de mercado son lo más consumido.

En este sentido, Marcos Pueyrredón, presidente del Ecommerce Institute, precisó que "el desafío de Ecuador es la profesionalización de la oferta, que permita enfrentar los volúmenes de compra y transacciones del comercio electrónico".

El ejecutivo comentó que, en el 2016, las compras en línea generaron casi US $\$ 900$ millones de dólares en el país y que para este año la cifra crecería un 50\%.

\section{COMPORTAMIENTO DE COMPRA}

Para iniciar con la definición del proceso de compra veremos primero que es el consumidor y lo podemos definir como "el conjunto de actividades que realizan las personas cuando seleccionan, compran, evalúan y utilizan bienes y servicios, con el objeto de satisfacer sus deseos y necesidades, actividades en las que están implicados procesos mentales y emocionales, así como acciones físicas" (Araujo Villa \& Fraiz Brea, 2011).

El proceso de compra no es sencillo, todo comprador pasa, consciente o inconscientemente, por diversas fases antes de ejecutar la compra de un producto, desde el momento en que se lo empieza a plantear por primera vez hasta que hace la compra.

Según el más reputado teórico del marketing mundial, Phillip Kotler, estas fases son cinco: reconocimiento de la necesidad, búsqueda de información, evaluación de alternativas, la decisión de compra, y el comportamiento poscompra. (Kotler \& Keller, 2006).

Recientemente, y ante la irrupción de la economía digital, se ha incorporado una nueva fase previa a las cinco de Kotler, que sería la de pre-contemplación.

La pre-contemplación sería la etapa en que los consumidores están en fase "durmiente": no han reconocido la necesidad de hacer una compra, ni siquiera la han contemplado todavía.

Es importante tener en cuenta esta etapa pues, pese a que los consumidores estén en fase "durmiente", el marketing puede actuar sobre ellos para despertarles la necesidad latente y no reconocida que llevarían dentro (Larripa, 2018).

El objetivo del marketing, en esta fase, seria desarrollar acciones que despierten a los consumidores y los lleven hacia la fase de reconocimiento de la necesidad.

\section{LA EXPERIENCIA DE MARCA}

Las funciones similares de los diferentes productos que muchas empresas ofertan al mercado implican una diferenciación de marcas cada vez más compleja, por lo que la utilidad del producto no es una característica competitiva y de ahí surge la necesidad de crear una mayor diferenciación de la oferta. 
El cliente no busca sólo información del bien o servicio que adquiere, sino la experiencia que puede experimentar. Esta experiencia se adquiere en cada uno de los contactos que se pueden tener con la marca. Para ello hay que entender qué es construir una "experiencia”.

Fernández y Delgado (2011) definen la experiencia de marca como un conjunto de respuestas internas subjetivas y de comportamientos suscitados o provocados por los estímulos relacionados con la marca que forman parte de su diseño, identidad, presentación, comunicación y entorno de venta” (Fernández Sabiote \& Delgado Ballester, 2011, p. 63).

Se puede decir que esta experiencia se forma como respuesta a la suma de cada interacción individual con la marca en cada uno de sus puntos de contacto y en cada uno de los estímulos directos e indirectos que se perciben de ella.

\section{IMPLICACIONES DE LA EXPERIENCIA DE MARCA}

En el caso de la implicación en la comunicación boca-oreja, está dada ya que este es un poderoso proceso de comunicación informal a través del cual se trasmite mucha información, y puede ser positiva y negativa y esta última implica un reforzamiento de esos atributos en mayor escala.

En Fernández y Delgado (2011) se dice que "la teoría sugiere que la comunicación boca-oreja es consecuencia de las respuestas emocionales a situaciones de consumo (Swean \& Oliver, 2000) que generan una tensión psicológica que puede verse aliviada cuando se comparten las experiencias con otros" (Westbrook, 1987).

Otra implicación es la identificación marca y consumidor que va más allá del intercambio meramente económico, pues está relacionada con aspectos de identidad personal y colectiva. Ahora bien, todas las marcas no tienen la capacidad de satisfacer esta dimensión y ello va a depender en gran medida del uso de herramientas de comunicación y otras que favorezcan la constante presencia de la marca en la vida del público objetivo.

Por último, es necesario abordar que la experiencia de marca depende en gran medida en la visión empresarial de trabajar unidos y en no relajar nunca la forma tratar a los consumidores a lo largo del tiempo, en todos los procesos desde la información hasta el momento de la compra, para no caer en promesas no cumplidas.

Es crucial entonces que en cada punto de contacto se creen procesos de retroalimentación con el consumidor final para generar una personalización de alta calidad y mejorar las experiencias de los consumidores y más en este mundo globalizado y dinámico donde el cliente busca no solo información del producto, sino también la experiencia que le proporciona la marca, desde el momento en que sabe de la marca en si ya sea porque oye hablar de ella o porque ve el anuncio de la misma, hasta que se decide probarla.

\section{METODOLOGÍA DE TRABAJO}

El enfoque de la investigación será mixto, ya que se utilizará tanto el cuantitativo, como el cualitativo, el cuantitativo se emplearán muestras representativas de sujetos, técnicas e instrumentos entre los que se encuentran los cuestionarios estructurados el enfoque cualitativo, según su visión será; interpretativa, de observación participante, entrevistas en profundidad o grupos focales entre otros, que permitirán la profundización de la información.

La primera técnica de investigación en utilizar fue el Grupo Focal, que para su realización se procedió a buscar perfiles de empresarios, docentes y personas que cumplieran con las características principales para desarrollar la investigación es así que se procedió a invitar a cuatro empresarios, dos docentes, dos personas de trabajo privado, dentro de la guía del moderador están los siguientes temas, bienvenida a los participantes, presentación del moderador y de cada uno de los integrantes, así como las preguntas de apertura, las de transacción y las de cierre. A continuación, detallamos los resultados:

1. Entre las plataformas que usualmente utilizan para realizar compras online se menciona en primer lugar, Amazon, por la confianza que depositan en ella, debido a su función legal y segura con los pagos a través de PayPal. Entre las plataformas internacionales destacan: Forever 21 (venta de prendas de vestir), Best Buy, eBay, AliExpress, Wish, Apple.

2. Entre las plataformas nacionales, que cabe destacar, no son plataformas de comercio 
electrónico como tal, sin embargo, son medios digitales que usan para satisfacer necesidades de compra están: Mercado Libre, la red social Facebook, OLX y plataforma para la compra de repuestos de vehículos.

3. Al solicitar que comenten sobre experiencia que hayan tenido al momento de realizar compras por internet, mencionaron lo siguiente:

- Entre las buenas experiencias que comentan esta la existencia de plataformas que tienen la certificación de los productos, las garantías correspondientes, y el cumplimiento en el tiempo de entrega, pues en la mayoría de los pedidos, incluso el producto llega antes de lo establecido, y que en algunos casos les mantenían informados y en forma constante en dónde se encontraba su pedido, desde el proceso de ensamble del computador hasta la misma entrega y recepción de este.

- Indican también, que han tenido buena experiencia al descubrir que, en algunas plataformas, Amazon, por ejemplo, se puede segmentar a los vendedores certificados de acuerdo al producto que se va a adquirir.

- Un punto de gran importancia es la facilidad y seguridad de pago a través de PayPal, tal es el caso de una ocasión en que no llegó el producto solicitado, luego del reclamo respectivo se realizó el reembolso de dinero por este medio.

- Entre las malas experiencias mencionan que en algunas plataformas los productos físicos no son iguales a los que se muestran en la fotografía, y tampoco brindan información de rastreo para saber dónde está la encomienda, en algunos casos llegó el producto.

- Otro aspecto importante que acotan es que, en algunos casos, en el certificado del producto no se especifican, ni se detallan todas las condiciones y características de este, como por ejemplo la existencia o no de repuestos; lo que complica la compra para un consumidor que considera esta característica indispensable al momento de la decisión de compra. Tal es el caso, de una plataforma en que, realizan la compra de un cargador portátil, y cuando llegó el producto, resulta que no funcionaba con batería sino con pilas, convirtiéndose así el producto poco apetecible para el uso que se esperaba.

- A pesar de que algunas plataformas reembolsan el dinero para los casos en los que el producto no llegó al destino, es importante recalcar también, que, de acuerdo con la experiencia, no en todos los casos es así, pues un participante comentó que el trámite de solicitud de reembolso se volvió complejo y tardado, dejando sin concluir este proceso.

- Haciendo alusión a plataformas nacionales, se dio un caso puntual, en donde la participante actuaba como vendedora, se confió de las buenas intenciones del comprador, recibiendo un cheque como pago a los productos entregados, cheque que resultó ser robado y obviamente ya no pudo localizar al comprador, perdiendo así su mercadería y dinero. En otro caso, se dio un anticipo por la compra realizada y jamás se recibió el producto.

4. Entre las características o atributos que consideran importante dentro de una plataforma virtual mencionan lo siguiente:

- Que la página posea una programación del sistema, que tenga certificaciones y que garantice un desarrollo seguro para el usuario, y de esta manera evitar clonaciones y ataques cibernéticos. En caso de existir dichas garantías, éstas deberían ser comunicadas al usuario final, pues al no hacerlo, la gente no confía en la plataforma y puede influir en su decisión de compra.

- La página debe ser dinámica, organizada y vivencial con el usuario, el diseño debería 
tener concordancia con un tiempo de respuesta rápida, se menciona que por ejemplo debería existir un chat o un buscador, con el objeto de mantener un contacto directo vendedor/usuario y viceversa.

- La plataforma debe ser accesible, de fácil búsqueda e identificación, especialmente para personas que no tienen mucha experiencia en compras online.

5. Lo que motiva a utilizar una plataforma online, al momento de realizar una compra es:

- La innovación del producto, de tal manera que sus características, que deben ser apreciables de manera digital, enamoren y se genere la compra.

- El ahorro de tiempo y la comodidad de seleccionar y recibir el producto sin tener que acudir a los almacenes de manera presencial.

- Los precios de plataformas internacionales, pues en la mayoría de los casos son más convenientes que los nacionales.

- La curiosidad por probar productos novedosos e interesantes que no se ven comúnmente en el país local.

- Las garantías que se presentan en las plataformas respecto al producto, enfocándose principalmente en las certificaciones que poseen los mismos.

6. Entre las plataformas virtuales locales, en donde los participantes han realizado compras y ha tenido una buena experiencia se menciona:

- Shopearlo, Almacenes La Victoria, Etafashion, Supermaxi.
- Es cierto que algunas personas han usado Mercado Libre, OLX, Facebook, y no han tenido inconveniente; pero en la mayoría de los casos, las experiencias con estas plataformas han sido negativas, pues consideran que no existe la seguridad, ni las garantías necesarias para efectuar la comprar, inspirando únicamente desconfianza; ya sea por el método de pago, por la veracidad de las características publicadas o por la forma de recibir el producto; pues, generalmente, se encuentra la publicación, se pone en contacto con el vendedor para solicitar las características específicas del producto, en caso de interesarle, se pacta día, lugar y hora para la entrega respectiva, situación, que en algunas ocasiones puede ser peligroso al no conocer la identidad real de dicha persona.

7. En cuanto a la percepción que tienen sobre las compras online, mencionan que la tendencia de hoy en día es el comercio electrónico, y que, de acuerdo con su punto de vista, la gente está abierta a realizar compras on line.

- Hablando específicamente del Ecuador, opinan que el país aún no está listo para manejar temas de plataformas completas y organizadas como Amazon, eBay, etc. Sin embargo, mencionan, que este tema, debería ser un reto para los empresarios del país, quienes deben investigar y tomar modelos de plataformas del exterior para poder plasmar esa cultura en el país y que los consumidores locales no recurran a plataformas ajenas.

- Generar un sentimiento de confianza en los consumidores en cuanto al uso de plataformas on line para realizar sus compras será duro, pues muchos de ellos al escuchar "comercio electrónico", lo asocian directamente con estafa, peligro, y cosas negativas. 
- La mejor manera de generar dicha confianza es conocer la experiencia que los demás han tenido con este proceso, de esta manera irá subiendo el número de consumidores que compren on line.

\section{INVESTIGACIÓN CUANTITATIVA}

Para realizar la investigación cuantitativa se eligió la técnica de la encuesta porque permite conocer estados de opinión de un segmento de mercado, y el nivel de satisfacción de las personas en un tema determinado o conocer anticipadamente la inclinación de los encuestados hacía un producto, marca y demás para tener información.

Para esta encuesta se tomó muy en cuenta a diferentes extractos demográficos, sociales y profesionales de la ciudad de Cuenca, para llegar a cumplir con el perfil adecuado de las personas que compran en tiendas online, el cual está enfocado a personas que poseen tarjetas de crédito, que tengan acceso a Internet, posean dispositivos electrónicos para realizar las transacciones, y la capacidad adquisitiva, además que conozcan el proceso del uso y forma de comprar y tengan necesidades de hacerlo en tiendas online.

Con la información anteriormente detallada se procedió a realizar las encuestas mediante un muestreo de 95 personas dividas en grupos de empresarios, estudiantes y profesionales, con la finalidad de cumplir con el perfil propuesto además de conocer cuál es el porcentaje de personas que utilizan plataformas online para realizar las compras y de conocer sus experiencias, hábitos y tiendas virtuales, que por lo general utilizan, así como qué les llama la atención de esas tiendas virtuales.

Las encuestas se realizaron en forma online mediante el google forms estas preguntas fueron enviadas a grupos de personas previamente identificadas mediante una base de datos obtenidas con grupos de empresarios de la mediana y pequeña industria, profesionales de las diferentes áreas, así como a un grupo de estudiantes de Instituciones Educativas Superiores.

El cuestionario para la investigación contó con 18 preguntas clasificadas en cerradas y abiertas, con la finalidad de saber cuáles son las mejores opciones de tiendas online o las más utilizadas, así como las recomendaciones para que se dé una mejor compra y uso de estas plataformas.

Entre el grupo de personas seleccionadas para la encuesta de la investigación se observó que existe un porcentaje del 55.8\% que es de sexo femenino y un $44.2 \%$ es de sexo masculino.

Podemos observar que la gran mayoría $66.3 \%$ de las personas que contestaron la encuesta está entre los 17 y 30 años que son por lo general de la generación $\mathrm{Z}$ y un $18.9 \%$ que en su gran porcentaje son los Millennials, estas dos generaciones son conocidas como nativos digitales y su información va a ser de gran ayuda para la propuesta de este proyecto.

Cuando se preguntó que, si realiza compras en internet, se trató de encontrar cuántas personas compran por internet lo cual nos dio como resultado que existe un $36.8 \%$ de las personas que no compran por internet lo que quiere decir que todavía se aprecia un rechazo por las compras online en la ciudad de Cuenca.

De este grupo de personas encuestadas y que no compran en internet, en su gran mayoría son mujeres (65.2\%) de la Generación $Z$ quienes dicen que han tenido malas experiencias cuando han realizado su primera compra, que no confían mucho en las plataformas de compra, que han tenido muchas dificultades con los productos por su mala calidad y por el tiempo de las entregas, por muchas vulnerabilidades y fraudes, que no generan la confianza para realizar las transacciones, y muchas de ellas porque no tienen la necesidad de comprar vía online, ya que prefieren comprar en forma personal.

El restante grupo de personas $34.78 \%$ son de las generaciones Millennials y de la generación X quienes también indican su mala experiencia en sus primeras compras, teniendo problemas como el producto no es el mismo al momento de recibirlo, que no existe la seguridad necesaria para entregar los datos de las tarjetas, y la demora en la entrega de los productos.

Continuando con el análisis de los resultados tenemos qué un $63.2 \%$ de los encuestados sí realizan las compras en internet, de este porcentaje existe un $37 \%$ de personas que son de la generación X y de los Millennials; y en su gran mayoría son de sexo masculino. 
De las personas que respondieron que sí efectúan las compras por internet se preguntó cuál es el uso que dan a esas compras, obteniendo como resultado que el 78.5\% las realiza para uso personal y que el $21.5 \%$ las realiza por cuestiones de negocios. $\mathrm{Al}$ analizar este $21.5 \%$ de personas que compran para uso en los negocios, podemos tener la siguiente información, los encuestados son de la generación $\mathrm{X}$ y Millennials, además podemos considerar que sus compras la realizan por más de seis veces al año, que utilizan por lo general tiendas virtuales como Amazon, Apple, EBay.

Entre los dispositivos electrónicos que utilizan están los teléfonos inteligentes, computadoras de escritorio y Tablet, los medios que manejan para traer sus productos en su gran mayoría son por Correos del Ecuador y Correos internacionales; sus compras sí han llegado en el tiempo establecido, todos han realizado el pago con tarjeta de crédito y por transferencia bancarias, que sí tienen la confianza en el uso de estos medios de pago que presentan esas tiendas online.

Califican la experiencia de compra como buena, y los productos que generalmente adquieren son ropa, y los artículos electrónicos; entre las características que toman en cuenta al momento de realizar sus compras está la seguridad de la plataforma y el fácil acceso, consideran que es ventajoso y rápido el realizar las compras en las plataformas online.

En su gran mayoría lo efectúan las transacciones por la noche y sólo un $33 \%$ de las personas no compran en tiendas virtuales locales, mientras que el resto de las personas lo realizan de tres a doce veces al año en estas, entre las recomendaciones que hacen para mejorar una Tienda Virtual, se encuentra que esta debe ser de fácil interacción, generar una entrega inmediata y a domicilio, tener confiabilidad y calidad de producto, poseer alianzas estratégicas con empresas de envíos.

Al examinar el $78.5 \%$ de los encuestados que realizan sus compras para beneficio personal, podemos señalar que de este porcentaje se tiene que el $67.3 \%$ son de las edades comprendidas de 17 a 30 años; y con un $52 \%$ son de sexo masculino y $48 \%$ son de sexo femenino, quienes en su gran mayoría han hecho compras de 1 a 3 veces al año, han comprado en tiendas virtuales como Amazon, Wish, EBay y Alibaba.
Entre los dispositivos electrónicos que han utilizado para efectuar las compras se encuentran los teléfonos inteligentes, y los medios para traer los productos a través de Correos del Ecuador; comentan en su gran mayoría que solo a veces cumplen los productos comprados con sus expectativas, hay solo un $18 \%$ que informa que el producto ha llegado a tiempo, el resto de los encuestados responde que solo a veces y que no han recibido a tiempo sus pedidos de compras.

Todas las personas han realizado sus transacciones mediante las Tarjetas de crédito y sólo una persona lo ha realizado por una cuenta de Paypal; dicen que no se sienten seguros ni confiados cuando brindan información personal para realizar la transacción de compra, todos expresan tener una buena experiencia al realizar la adquisición a excepción de una persona que señala haber tenido una mala experiencia de compra. Entre los productos que han adquirido mediante las tiendas virtuales están la ropa, electrodomésticos, juguetes y accesorios.

Entre las características o atributos que toman en cuenta para realizar la compra está como primer punto la seguridad de la plataforma, el aspecto visual o diseño de la web, y el fácil acceso a la plataforma, además consideran muy atractivo, rápido, ventajoso, obsesionante y complejo realizar las compras en internet, señalan que han utilizado hasta tres veces al año las tiendas online locales, solo dos personas indican que nunca lo han realizado, el horario más conveniente para realizar las adquisiciones para este segmento es en la noche.

Entre las sugerencias que indican para mejorar las tiendas virtuales están: Que exista una información más detallada de los productos así como de una mayor variedad, deben reducir los pasos al momento de finalizar la compra, que los productos sean de calidad y que los productos que entregan sean como los que ofrecen en las tiendas, demostrar mayor seguridad y confiabilidad, que exista un espacio para dejar las opiniones, sugerencias y reclamos, así como el cumplimiento de los tiempos de entrega.

En relación con la pregunta de la experiencia de compra podemos observar que un $81 \%$ de los encuestados que contestan la califican de buena, en su mayoría son de las generaciones Millenials y Z, y las plataformas que han

utilizado son Amazon, Apple, los productos que más han adquirido son ropa y accesorios electrónicos. 
Un $14.3 \%$ cree que es excelente, y son las personas que por lo general realizan la compra más de cuatro veces por año, que lo han realizado por Amazon y por Ebay, además indican que ha llegado a tiempo el producto comprado, además han utilizado tarjetas de crédito, estas personas el atributo que toman en cuenta en el momento de realizar las compras es la seguridad, y han comprado accesorios electrónicos, ropa y juguetes.

El restante $4.8 \%$ la califican de mala y son los que han usado la plataforma Wish y han traído por los Correos del Ecuador.

\section{DISCUSIÓN Y RESULTADOS}

Luego de haber realizado la recopilación de información tanto bibliográfica como de las técnicas de investigación (grupo focal, y encuestas), y de conocer como es el comportamiento de las personas de la ciudad de Cuenca en relación con sus experiencias de compra en plataformas online, se propone las siguientes alternativas fundamentadas en el neuromarketing y en la implementación de los sentidos, para el mejoramiento del diseño, atracción de los clientes, e implementación de las tiendas virtuales en el medio local con la finalidad de ayudar a los empresarios, emprendedores y los de las pequeñas y medianas industrias del sector a incrementar las ventas, posicionamiento y aceptación de los productos que comercializan.

Los estudios de neuromarketing son instrumentos que nos ayudan a decodificar lo que los consumidores piensan al estar delante de un producto o una marca, y a descubrir cuáles son los métodos que emplean los profesionales del marketing para seducirnos sin nuestro conocimiento.

Según Roger Dooley en su obra Brainfluence, nos comenta sobre los diferentes aspectos sensoriales que deben ser considerados como estímulos relevantes para el consumidor. Específicamente en el sentido visual, existen condiciones que deben ser debidamente cuidadas, como por ejemplo el tipo de letra, el mismo que debe ser sencillo, para facilitar la tarea de lectura. Además, un tipo de letra sencillo incita a la acción. (Dooley, 2015), si se quiere que alguien complete un formulario, las instrucciones deben ser cortas y estar escritas en un tipo de letra sencillo.

Fundamentados en la investigación realizada así como en la información de los autores se propone que una tienda virtual tiene que estar construida en base a la estimulación de los sentidos por eso se plantea los siguiente pasos para la creación y diseño de una tienda virtual para la implementación en los negocios de la ciudad de Cuenca; esta propuesta se basó en un artículo por parte del profesor argentino Adolfo Ariza, titulado "Como crear una tienda on line en diez pasos, tres meses y sin inversión” (Ariza, 2019), quien presenta una estructura fácil y sencilla, basado en la comodidad y accesibilidad de encontrar la información en internet $y$ de las diferentes aplicaciones y estrategias tecnológicas.

\section{PASOS PARA LA CREACIÓN DE UNA TIENDA VIRTUAL}

\section{Escoger el nombre y el dominio de tu tienda online:}

Muchas veces los nombres que queremos poner a un negocio ya están utilizados o registrados por eso es muy importante, tener un nombre que esté relacionado con la actividad comercial, de fácil recordación, además debe ser corto, sencillo, atractivo, llamativo, duradero e innovador, el primer desafío siempre debe ser pasar de tener sólo un nombre a tener una marca; las grandes empresas han pasado de tener un nombre, a ser un concepto de marca y esto se logra con actividades y estrategias de marketing ya que una marca pasa a ser mucho más que un isotipo o un logotipo. Si ya tienes tu propia marca o empresa registrada, el procedimiento es más fácil.

El nombre tiene que estar muy relacionado con la elección del dominio, a modo de resumen se dan las siguientes consideraciones: Comprar tanto el dominio (.com) como el dominio del país (.ec). No supone apenas coste adicional y te aseguras de proteger tu marca frente a la competencia.

Hay que elegir un dominio fácil de recordar y pronunciar, por lo general no hay que confundir a los clientes con términos en inglés, la elección de un buen dominio es fundamental en la creación de una tienda online y para su posicionamiento. Es muy importante que dentro del nombre y del dominio estén algunas palabras claves que puedan ayudar a estar presentes en los buscadores de internet.

\section{Elige una buena empresa de hosting}


Esta es prácticamente la única inversión que se deberá realizar para crear la tienda online, así que hay que efectuar la mejor elección y no fijarse en lo más barato, por eso es preferible buscar una empresa que ofrezca un hosting de calidad y garantía.

La velocidad de una tienda online es clave, tanto para los usuarios como para el posicionamiento en los buscadores. Una página lenta hace que los usuarios se cansen de esperar y la abandonen.

Hay que asegurarse de que la empresa de hosting garantice el espacio suficiente para la tienda online y una buena velocidad de conexión. Contratando un buen servicio de hosting se evitará también caídas innecesarias del servidor y se podrá tener una tienda disponible 24 horas al día, los 365 días al año.

\section{Personaliza tus productos}

Muchas plataformas de mayoristas proveen a las tiendas online de su catálogo de productos en formatos que pueden ser subidos a tu tienda automáticamente, y así se pueden mostrar todos los productos en muy poco tiempo. Pero es básico personalizar todos los textos de la tienda.

A la larga los únicos productos que se posicionarán correctamente serán aquellos que hayas modificado, y los usuarios agradecerán no encontrar el mismo texto que verán en otras tiendas. Amplía la información con detalles del producto, fotos, métodos de uso o de funcionamiento, hay que usar mucho la imaginación.

Es muy importante utilizar fotografías que llamen la atención de los consumidores como realizar un catálogo interactivo, con colores que cautiven al público objetivo, imágenes en movimiento y con la información clara y precisa, se puede presentar imágenes de los productos en pantalla completa y desplegable.

\section{Añade todos los métodos de pago posibles}

Es imprescindible ofrecer el máximo de métodos de pago posibles en tu tienda online. Todavía hay gente que no se fía de comprar por Internet, y solamente realiza pedidos a tiendas que ofrecen el método contra pedido, ya que sólo pagará al recibir el paquete. Otros, siempre utilizan las tarjetas ya que entienden que detrás habrá una empresa y algunos utilizan transferencia bancaria porque les es más cómodo. Se pueden generar transferencias bancarias, hay que tener presente el porcentaje de cobro que se realizará por cada una de las transacciones.

Paypal pone a disposición una pasarela de pago que permitirá cobrar a nuestros clientes con tarjeta. Su instalación es sumamente sencilla y el único coste que va a tener para la empresa será la comisión que nos cobrará Paypal por cada transacción realizada.

\section{5.- Promociona tu tienda online}

Al tener nuestra tienda online configurada, nuestro catálogo instalado y todo listo para empezar a vender, ahora el siguiente paso será buscar la forma para que nos encuentren en el difícil mundo de Internet ya que las visitas no llegarán solas a tu web, es fundamental poner en marcha todas las áreas del marketing digital para conseguir atraerlas a la tienda online y hacerlas que compren, es por eso que se plantean que se realicen las siguientes estrategias:

- $\quad$ SEO o posicionamiento en Google, consigue aparecer en los primeros resultados de las búsquedas relacionadas con tus productos, busca palabras claves que estén relacionadas con tu empresa, o producto. Hay que definir una estrategia en redes sociales, hay que recordar que no hay que estar en todas, sino en aquellas donde se encuentra tu público objetivo.

- Un blog te ayudará a conseguir más visitas y comercializar tus productos desde los artículos que publiques. Buscar influencers de tu sector para darte a conocer y mejorar tus ventas. SEM o publicidad online, hay que invertir periódicamente en anuncios de publicidad para dar a conocer tu tienda online y lanzar campañas de promoción de tus productos.

\section{Incluir aspectos legales dentro de la tienda online}

Una tienda online está sujeta a una serie de temas legales que se deben cumplir, entre otros: Reglamento General de Protección de Datos, Ley de Cookies, Leyes de Comercio Electrónico. 
De no hacerlo, te pueden llevar a una multa considerable, entre algunas de las recomendaciones es que no hay que copiar los textos legales de la tienda online parecida ya que puede que esté desactualizada y que no cumpla con las normativas legales del sector o región, es preferible acudir a abogados expertos en nuevas tecnologías para asegurarte de que cumples con la legalidad.

Hay que asegurarse de tener todos los papeles en regla, contratar un seguro, y cumplir con todas las leyes vigentes, una multa o sanción económica por no actuar correctamente podría hacer desaparecer tu empresa y tu proyecto.

\section{7. -Crea campañas de publicidad online}

Gracias a la publicidad online, podemos dar a conocer nuestra tienda online de forma económica, pero sobre todo de forma progresiva. Usando correctamente las herramientas de análisis gratuitas que Internet pone a nuestra disposición, podremos saber con exactitud qué campañas publicitarias nos dan beneficios y cuáles no funcionan, pudiendo rectificar rápidamente y evitando pérdidas innecesarias.

Para empezar a publicitarse en internet, es recomendable usar la publicidad en los resultados de búsqueda de Google mediante Adwords, otro aspecto importante es utilizar la publicidad off line, manejando los medios de comunicación tradicionales y medios ATL y BTL que es de gran utilidad para lograr posicionar en el mercado objetivo al que me quiero dirigir.

\section{RECOMENDACIONES PARA REALIZAR UNA TIENDA VIRTUAL MÁS LLAMATIVA E INNOVADORA}

A continuación, se va a detallar algunos ejemplos que se pueden utilizar para diseñar una tienda virtual atractiva e innovadora basada en los sentidos y en los estudios de neuromarketing y que han dado resultados en algunas empresas a nivel mundial.

Utilizar una tipografía y un diseño agradable a la vista, incluyendo potentes opciones de filtrado y controles deslizantes que permiten al usuario interactuar y ajustar la compra a sus necesidades.
Una estrategia muy útil que se ha aplicado en la versión para móviles es en la que vibra el teléfono cuando un producto se ha añadido al carrito de compras, como un indicador de que se ha añadido correctamente el producto, esto genera una interacción entre el cliente y la tienda.

Una estrategia que puede servir para mejorar la experiencia del cliente en boutiques es que se puede permitir a este escoger grupos de productos para configurar un atuendo completo. De este modo, que mejoran la experiencia por personalizar su atuendo y además se generan pedidos mayores al vender los productos en conjunto.

Otra estrategia de innovación en la experiencia del usuario se puede centrar en el sentido de la vista, ya que se puede introducir un fondo animado de la naturaleza en su página de inicio, pretendiendo transmitir la sensación de ser parte de la concientización ambiental

Las secciones de Ayuda en las tiendas virtuales son, también, un aspecto fundamental para mejorar la experiencia de compra ya que, en estas secciones, el usuario puede encontrar toda la información acerca de los productos disponibles, las mejores formas de buscarlos, las condiciones legales, las políticas de seguridad, la forma de tramitación y de pago de los pedidos, etc.

La usabilidad es un factor importantísimo que repercute directamente en la experiencia de compra en una tienda virtual; por ello, no solo hay que tenerlo muy en cuenta a la hora de crear cualquier página web o aplicación móvil sino, también, cuando queremos abrir una tienda en internet.

Poner el botón "comprar" en una tienda online está generando la usabilidad de la página, es que, si este botón no está a la vista y el proceso de compra no es rápido, al final se realizarán menos compras y entrarán menos usuarios a la tienda virtual, es por eso aconsejable poner el botón de compra en la primera página o tener una opción en su web que diga "para comprar en un solo clic", lo que supone una solución muy efectiva al momento de tomar la decisión de compra.

Una tienda virtual puede cobrar vida con animaciones que se entrelazan con los elementos de la tienda, como, por ejemplo, 
copos de nieve cayendo, chimeneas llameantes, imágenes tipo gifs, relacionadas a los productos o servicios que comercializa. Además, se puede utilizar la imagen como punto de acceso a las categorías o productos mediante un click, Se puede también utilizar el puntero de su ratón para girar los productos.

Una estrategia que ha funcionado mucho es promocionar o generar tiendas virtuales en áreas públicas con alto tráfico peatonal, como estaciones de bus, donde los clientes podían usar una aplicación móvil, gratis o realizando el escaneo del código QR para que generen sus pedidos de productos mientras espera su transporte, además puede programar su entrega.

Las redes sociales pueden ser una mina de oro para incrementar el conocimiento sobre el consumidor y las oportunidades de engagement (atracción de los clientes), ya que se puede generar información acerca de los beneficios y utilidades de sus productos. Además, con las herramientas de escucha social que permiten la recopilación y agregación de datos en tiempo real, se puede tener la capacidad de reaccionar y optimizar las estrategias de experiencia del cliente con mayor velocidad.

\section{CONCLUSIONES}

Luego de recolectar y analizar diferentes puntos de vista, opiniones y experiencias, se concluye que, en la actualidad, la necesidad de encontrar un medio más efectivo para la satisfacción de necesidades ha permitido que se usen medios digitales, para que de esta manera la búsqueda, comparación, selección y pago de productos, se realicen de manera rápida, ahorrando así tiempo y recursos.

Se han determinado hábitos y características más comunes que los consumidores muestran al momento de decidir si la compra de sus productos será de forma on line, quienes, en primer lugar, toman en cuenta, que la plataforma sea dinámica con el usuario, de tal manera que el producto elegido sea el correcto y al realizar la compra se sienta la satisfacción que se esperaba. Otro aspecto importante son las certificaciones del producto y la seguridad que proporcione la plataforma, pues hoy en día los avances tecnológicos han permitido que los "delincuentes" aprovechen estas ventajas para actuar de manera negativa y perjudicar a la sociedad.
Esta publicación forma parte del proyecto de investigación titulado: Influencia de la experiencia de marca en consumidores de compra online en la ciudad de CuencaEcuador, financiado por el Instituto Tecnológico Superior Sudamericano. De la investigación obtenida se puede indicar que el comportamiento del consumidor cuencano es que son muy inseguros para el uso de las plataformas online pero que una vez experimentado positivamente el proceso de compra online se vuelven más confiados para poder realizar más compras continuamente; no así las personas que han desarrollado una mala experiencia por algunos problemas de entrega o por la percepción de los productos es difícil que quieran volver a realizar la compra.

Muchos de los futuros usuarios de las compras online en la ciudad de Cuenca son los de la generación Z, por lo cual hay que prestar mucha atención en el comportamiento de este segmento para poder generar estrategias que vayan encaminadas a satisfacer las necesidades y deseos de esta generación, que por lo general entre una de sus características son las de que prefiere comprar en las tiendas virtuales, porque les es más fácil y cómodo comprar a través de una computadora que ir a la tienda por un artículo.

Al poner en práctica los estudios de neuromarketing que han sido ya comprobados en algunas tiendas virtuales a nivel global, puede ser de gran ayuda para los negocios locales, así ser más competitivos; y para generar mayores ingresos.

Finalmente, en Ecuador, ninguna empresa aún ha generado una plataforma similar a las internacionales, es por esto, que los consumidores acuden a Amazon, eBay, Best Buy, entre otras; dichas experiencias han permitido dar el primer paso en definir guías didácticas para que los estudiantes, especialmente de la carrera de marketing, conociendo las necesidades que tienen los consumidores en temas de compras on line, logren determinar estrategias óptimas para satisfacer las mismas y posicionar marcas.

De igual manera, el empresario permitirá analizar y generar nuevas rutas y métodos de compra, para que así, las necesidades demandadas sean solventadas localmente. 


\section{REFERENCIAS BIBLIOGRÁFICAS}

Araujo Villa, N., \& Fraiz Brea, J. A. (2011). Comportamiento y perfil de los consumidores de series de ficción. Vivat Academia, 47-62.

Google Scholar

Ariza, A. (08 de abril de 2019). www.kusca.com.ar. Obtenido de https://www.kusca.com.ar/como-crear-unatienda-on-line-en-diez-pasos-tres-meses-y-sininversion/

Dooley, R. (2015). Brainfluence. Barcelona: Empresa Activa.

El Universo. (2017, 08 de noviembre). www.eluniverso.com. Obtenido de https://www.eluniverso.com/noticias/2017/1 1/08/nota/6469216/estudio-revela-habitoscompra-internautas

Electrónico, C. E. (2017). http://www.cece.ec. Obtenido de https://drive.google.com/file/d/1zA1A8R3yq-2dv2qztMx18Z8afERgldi/view

Fernández Sabiote, E., \& Delgado Ballester, M. (2011). Marcas de Experiencia: Marcando la diferencia. Estudios Gerenciales, 27(121) 59-77. DOI: https://doi.org/10.1016/S0123-5923(11)70181-4

Google Scholar Scopus

Kotler, P., \& Keller, K. (2006). Direccion de Marketing. Mexico: PEARSON.

Larripa, S. (2018, mayo). cuadernodemarketing.com. Recuperado de https://cuadernodemarketing.com/conoce-las-5fases-del-proceso-de-compra-para-vender-mejorl

Lindstrom, M. (2012). Buyology. verdades y mentiras de por qué compramos. Barcelona: Ediciones Gestion.

Porter, M. (2015). Ventaja Competitiva. Mexico: Patria.

Schmitt, B. (2000). Experiential Marketing. Barcelona: DEUSTO.
Steenkamp, J. B. E., \& Baumgartner, H. (2000). On the use of structural equation models for marketing modeling. International journal of research in marketing, 17(2-3), 195-202.

\section{Google Scholar Scopus}

Westbrook, R. A. (1987). Product/consumption-based affective responses and postpurchase processes. Journal of marketing research,24(3), 258-270.

\section{Google Scholar}

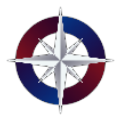

